\title{
mTOR inhibitors, a new era for metastatic luminal HER2-negative breast cancer? A systematic review and a meta-analysis of randomized trials
}

\author{
Maria Saveria Rotundo, Teresa Galeano ${ }^{1}$, Pierfrancesco Tassone ${ }^{2,3}$, Pierosandro \\ Tagliaferri $^{1}$ \\ ${ }^{1}$ Department of Experimental and Clinical Medicine, Medical Oncology, Magna Graecia University, Viale Europa, and \\ Catanzaro, Italy \\ ${ }^{2}$ Department of Experimental and Clinical Medicine, Translational Medical Oncology, Magna Graecia University, Viale \\ Europa, Catanzaro, Italy \\ ${ }^{3}$ Sbarro Institute for Cancer Research and Molecular Medicine, College of Science and Technology, Temple University, \\ Philadelphia PA, USA
}

Correspondence to: Pierosandro Tagliaferri, e-mail: tagliaferri@unicz.it

Keywords: metastatic breast cancer, luminal breast cancer, meta-analysis, hormonal therapy, mTOR inhibitor

Received: November 02, $2015 \quad$ Accepted: January 29, $2016 \quad$ Published: February 17, 2016

\section{ABSTRACT}

We evaluated if standard hormonal therapy (HT) could be improved by the addition of mammalian target of rapamycin inhibitors (MTOR-I) in metastatic luminal breast cancer. A meta-analysis on 4 phase II-III randomized clinical trials was performed. Pooled hazard ratio (HR) for progression free survival (PFS)/ time to progression (TTP) was 0.62 in favor of $\mathrm{mTOR}-\mathrm{I}+\mathrm{HT}$ arm (95\% confidence interval [CI] $0.55-0.70 ; p<0.0001)$. There was significant heterogeneity for PFS/TTP (Cochran's $Q 32, p<0.0001, I^{2}$ index $90.6 \%$ ). Pooled HR for overall survival (OS) was 0.84 in favor of the combination arm (95\% CI 0.71-0.99; $p=0.04)$. Heterogeneity was not significant (Cochran's Q 4.47, $p=0.1, \mathrm{I}^{2}$ index 55.3\%). Pooled risk ratio (RR) for objective response rate (ORR) was 0.88 in favor of experimental arm (95\% CI 0.85$0.91 ; p<0.0001$ ). Heterogeneity was not significant (Cochran's $Q 2.11, p=0.3, I^{2}$ index $5.2 \%$ ). Adverse events (AEs), in particular those of grade 3-4, mostly occurred in mTOR-I+HT arm. Combination therapy of HT plus MTOR-I improves the outcome of metastatic luminal breast cancer patients. Our results provide evidence of a classeffect of these targeting molecules.

\section{INTRODUCTION}

In the last decade the development of new therapeutic approaches for luminal breast cancer [1] has been an active area of investigation, since this molecular subtype could indeed represent a stand-alone class in breast cancer scenario [2]. In this context, specific targeting of mTOR, a molecular checkpoint involved in cell proliferation and immune microenvironment modulation, is a promising approach to improve the current treatment [3-4].

On this basis, we performed a systematic review on randomized trials investigating mTOR-I in combination to HT as compared to HT alone in metastatic luminal breast carcinoma. Subsequently, we conducted a meta-analysis to determine benefit and safety of the combined treatment.
The aim of our study was to investigate if the addition of mTOR-I to standard HT could produce a class-effect on luminal breast cancer outcome.

\section{RESULTS}

Studies selection

Three-hundred nineteen articles from MEDLINE bibliographical database were found. All no comparative studies, no randomized clinical trials, studies that not involved our target drugs were excluded. The remaining 87 articles were further reviewed and only 6 articles met our inclusion criteria. The searching and selection process is outlined in Figure 1. 


\section{Individual studies characteristics and results}

All studies were conducted on postmenopausal women with hormone receptor positive and HER2negative advanced breast cancer. One phase II trial evaluated everolimus plus tamoxifen versus tamoxifen alone (TAMRAD). One phase III trial evaluated everolimus plus exemestane versus exemestane alone and the results are reported in 3 articles (BOLERO-2 trial). One phase III trial evaluated temsirolimus plus letrozole versus letrozole (HORIZON). One phase II trial evaluated sirolimus plus tamoxifen versus tamoxifen (Bhattacharyya trial). The included studies were published between 2011 and 2014. The total number of patients from all trials was 2147 . The characteristics and efficacy results of the selected studies are reported in Table 1.

\section{Everolimus}

TAMRAD phase II randomized study investigated the efficacy and safety of everolimus $10 \mathrm{mg}$ daily plus tamoxifen $20 \mathrm{mg}$ daily versus tamoxifen alone in aromatase inhibitor (AI) resistant breast cancer patients. TTP (secondary endpoint) was 8.6 months in experimental arm versus 4.5 months in control arm (HR: 0.54; $95 \%$ CI $0.36-0.81 ; p=0.0021)$. A $55 \%$ reduction in risk of death was achieved in combination arm (HR: 0.45 ; $95 \%$ CI $0.24-$ $0.81 ; p$ exploratory $=0.007$ ). ORR was $14 \%$ in tamoxifen plus everolimus and $13 \%$ in tamoxifen alone groups, respectively. Most common AEs in the combination group were stomatitis, fatigue, rash, diarrhea and anorexia [5].

In BOLERO-2 phase III randomized trial everolimus $10 \mathrm{mg}$ daily plus exemestane $25 \mathrm{mg}$ daily was compared to exemestane alone in postmenopausal women with hormone
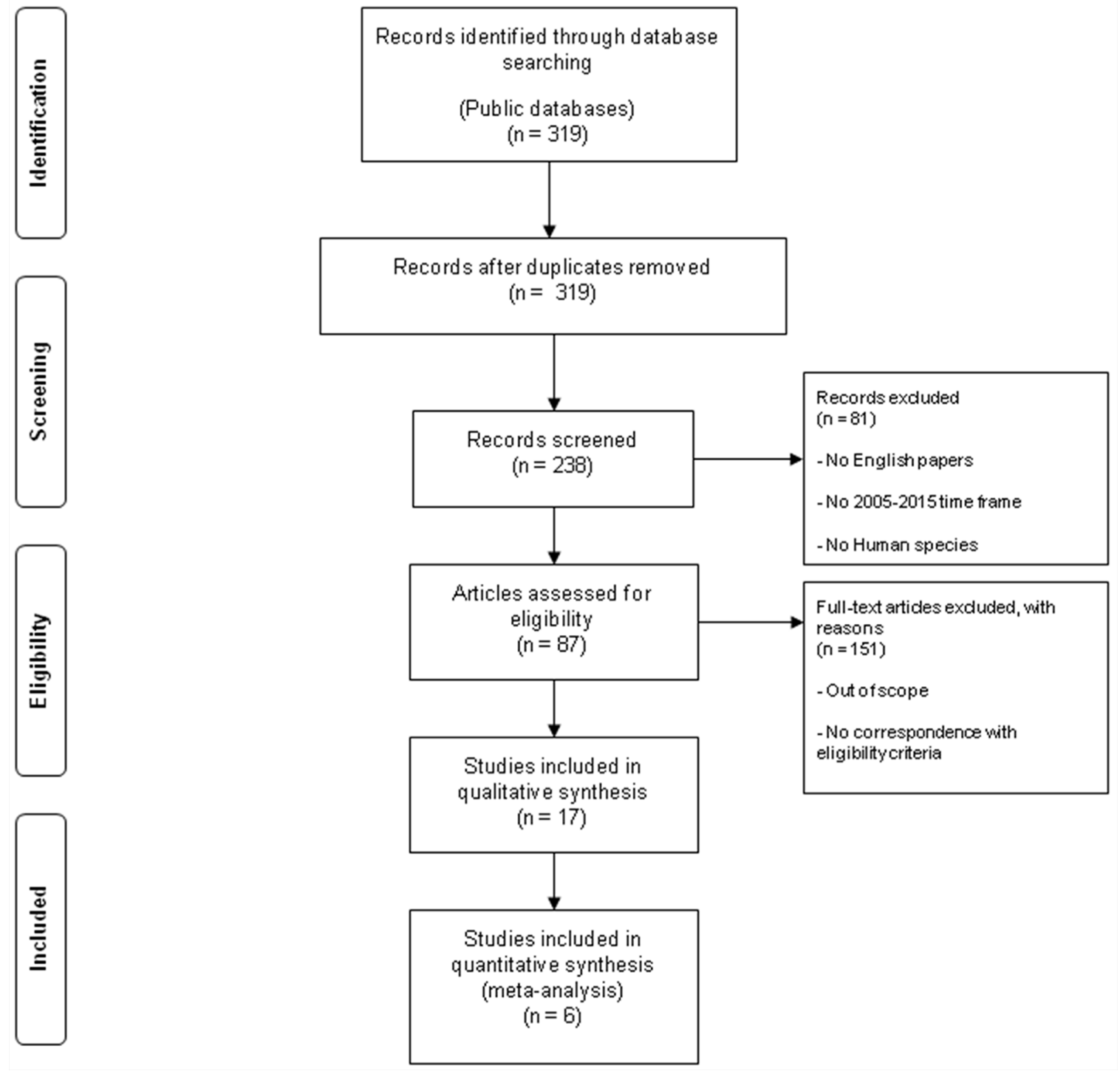

Figure 1: Flow chart of the literature search used in this meta-analysis. Public databases (PubMed, Embase, Central Registry of Controlled Trials of the Cochrane Library) full texts and abstracts were performed to track relevant references for the 2005-2015 time frame. 
Table 1: Characteristics and efficacy results of the eligible studies

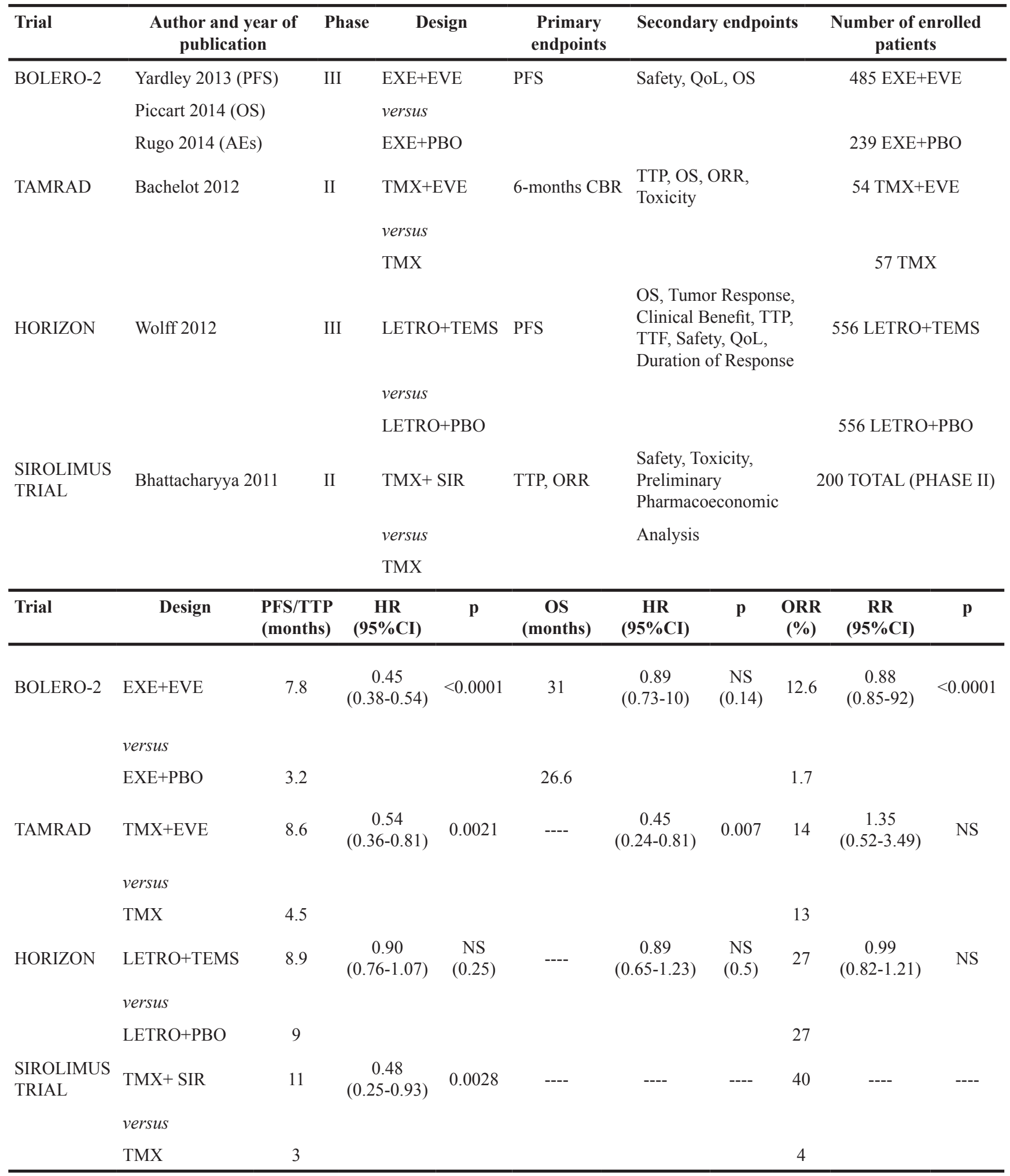

EXE: exemestane, EVE: everolimus, PBO: placebo,TMX: tamoxifene, LETRO: letrozole, TEMS: temsirolimus, SIR: sirolimus, PFS: progression free survival, CBR: clinical benefit rate, TTP: time to progression, ORR: objective response rate, QoL: Quality of Life , OS: overall survival, TTF: time to treatment failure, NS: not significant. 
receptor positive HER2-negative advanced disease recurred or progressed after treatment with letrozole or anastrozole [6]. The median PFS (primary endpoint) was 7.8 months in combination therapy arm (485 patients) versus 3.2 months in control arm (239 patients) (HR: 0.45; 95\% CI $0.38-0.54 ; p<0.0001)$ [7]. In post hoc analysis median postprogression survival in patients who had progressed at the time of the final PFS analysis was similar for both arms (20.8 months, 95\% CI 17.3-23.3, versus 19.3 months, 95\% CI 15.9-23.9). Final OS was 31 months (95\% CI 28.0-34.6) in combination arm (482 patients) versus 26.6 months $(95 \%$ CI 22.6-33.1) in HT alone (238 patients) (HR: 0.89; 95\% CI $0.73-1.10 ; p=0.14$ ) [8]. ORR was significantly higher for the combination therapy versus HT alone (12.6\% versus $1.7 \%$; $p<0.0001$ ) [7]. AEs mostly reported in experimental arm were pneumonitis, stomatitis, rash, dyspnea, fatigue [9]. The most common grade 3-4 AEs were reported in everolimus plus exemestane arm, with 22 deaths in combined arm and 4 deaths in placebo plus exemestane [8].

\section{Temsirolimus}

After a promising phase II trial on temsirolimus 30 mg daily for 5 days every 2 weeks and letrozole $2.5 \mathrm{mg}$ daily versus letrozole alone, in postmenopausal women with recurrent or metastatic disease [10], the combination treatment was investigated in the phase III HORIZON, in postmenopausal hormone receptor positive women not treated with AI, with advanced or metastatic disease. The primary endpoint PFS resulted similar in both groups (HR: $0.90 ; 95 \%$ CI $0.76-1.07 ; p=0.25)$ and no differences in OS (HR 0.89; 95\% CI 0.65-1.23) and in ORR (RR=0.99; 95\% CI 0.82-1.21) were observed. Any grade and grade 3-4 AEs (stomatitis, diarrhea, rash, hyperglycemia) were mostly reported in the experimental arm [11].

\section{Sirolimus}

In a phase I-II trial patients who could not afford AI were randomized to tamoxifen $20 \mathrm{mg}$ daily or tamoxifen plus sirolimus $2 \mathrm{mg}$ daily and patients who had failed AI and/ or tamoxifen were also randomized to the combination. In the phase II trial the primary endpoint TTP was improved by 3.3 months to 11.7 months adding sirolimus (HR: 0.43 ; 95\% CI $0.25-0.92 ; p=0.0023$ ) in patients progressed during treatment with prior AI or tamoxifen. In patients who had not received AI, sirolimus plus tamoxifen improved median TTP of 7 months compared to tamoxifen alone (HR 0.48; 95\% CI 0.25-0.93; $p=0.0028$ ). The most common AEs in experimental arm were hyperglycemia, hypercholesterolemia, hypetriglicerydemia, stomatitis, rash and anorexia [12].

\section{Meta-analysis results}

\section{Efficacy}

We evaluated PFS/TTP, OS and ORR to establish efficacy of mTOR-I+HT arm versus HT arm. Pooled HR for PFS/TTP, performed combining all the 4 trials, was 0.62 in favor of mTOR-I+HT arm (95\% CI 0.55-0.70; $p<0.0001)$ at fixed-effects model analysis. There was significant heterogeneity for PFS/TTP (Cochran's Q 32, $p<0.0001, \mathrm{I}^{2}$ index 90.6\%). Pooled HR for PFS/TTP was 0.58 in favor of mTOR-I+HT arm (95\% CI 0.37-0.90; $p=0.01$ ) at random-effects model analysis, too (Figure 2). The heterogeneity was still detected for PFS/TTP when Bhattacharyya trial was removed from the analysis (fixedeffects pooled HR 0.63; 95\% CI 0.56-0.71; $p<0.0001$; Cochran's Q 31.3, $p<0.0001, \mathrm{I}^{2}$ index 93.6\%; randomeffects pooled HR $0.60 ; 95 \%$ CI $0.36-1.00 ; p=0.05$ ) (Figure 3).

Pooled HR for OS, performed excluding Bhattacharyya trial, was 0.84 in favor of the combination arm (95\% CI 0.71-0.99; $p=0.04)$. Heterogeneity was not significant (Cochran's Q 4.47, $p=0.1, \mathrm{I}^{2}$ index 55.3\%) (Figure 4).

Pooled RR for ORR, performed excluding Bhattacharyya trial, was 0.88 in favor of experimental arm (95\% CI 0.85-0.91; $p<0.0001)$. Heterogeneity was not significant (Cochran's Q 2.11, $p=0.3, \mathrm{I}^{2}$ index 5.2\%) (Figure 5).

\section{Safety}

Data for each AE were extracted in those trials reporting them consistently. The pooled RR for AEs was performed using the fixed-effects model analysis. A random-effects model analysis was added for a more conservative estimate in the presence of heterogeneity. The $\mathrm{I}^{2}$ index has been evaluated in addition to Cochran's Q. Any grade diarrhea, pneumonitis, infection and dyspnea resulted significantly more frequent in the mTOR-I+HT arm and the heterogeneity did not show significant difference at Cochran's Q test. However for diarrhea the $\mathrm{I}^{2}$ index was high $(73.4 \%)$, while for pneumonitis, infection and dyspnea the $\mathrm{I}^{2}$ index was medium (39.7\%), medium (55.3\%) and low (0\%), respectively. Any grade asthenia, fatigue, stomatitis and rash were significantly more frequent in the experimental arm, but the heterogeneity was significant at Cochran's $\mathrm{Q}$ test and it was confirmed by $\mathrm{I}^{2}$ index. Asthenia and fatigue did not show statistical differences between arms at random-effects model analysis. Hyperglycemia did not show differences between arms, too, but significant heterogeneity resulted at Cochran's Q test and at $\mathrm{I}^{2}$ index. Grade 3-4 asthenia, fatigue, diarrhea, pneumonitis, rash and dyspnea significantly resulted more frequent in the mTOR-I+HT arm and the heterogeneity did not show significant difference at Cochran's $\mathrm{Q}$ test $\left(\mathrm{I}^{2}\right.$ index: 0\% for asthenia, diarrhea, pneumonitis and dyspnea, $6.3 \%$ for fatigue, $64 \%$ for rash, respectively). Grade 3-4 infection resulted similar in both arms and the heterogeneity did not show significant difference at Cochran's Q test, with a low $\mathrm{I}^{2}$ index (0\%). Grade 3-4 stomatitis and hyperglycemia 
resulted significantly higher in combination arm at fixedeffects model, but not at random-effects model analysis, with a significant heterogeneity at Cochran's Q test and an $\mathrm{I}^{2}$ index of $68.4 \%$ for stomatitis and $84.8 \%$ for hyperglycemia, respectively (Table 2).

\section{Publication bias}

A funnel plot was drawn for PFS/TTP and no asymmetry was detected. P value was 0.33 at BeggMazumdar test and 0.77 at Egger test, respectively, providing statistical evidence of funnel plot symmetry and then of the absence of publication bias $(p>0.05)$. These tests cannot perform when $>3$ trials data are not available. So we could not check meta-analysis biases by the other outcomes (Figure 2).

\section{DISCUSSION}

In the light of the most recent advances, the standard of care of metastatic luminal breast cancer is nowadays
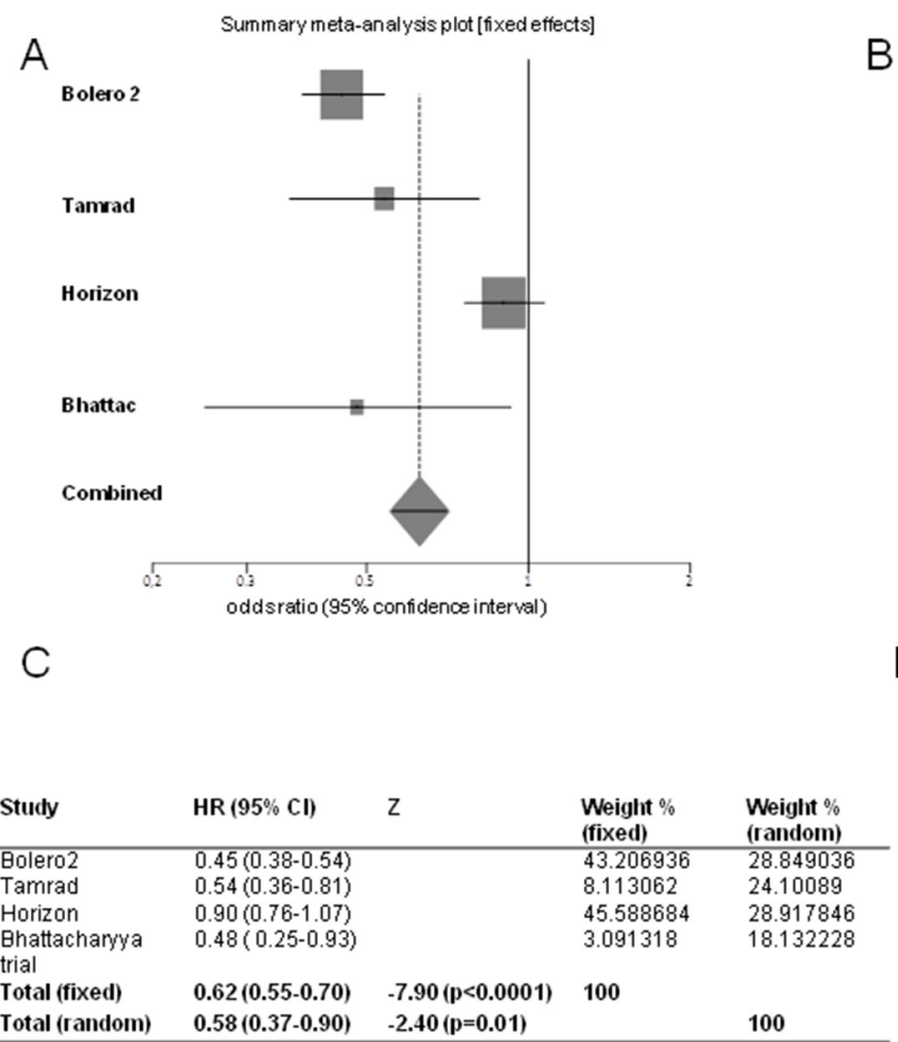

Total (random) $\quad 0.58(0.37-0.90) \quad-2.40(p=0.01)$

Cochran $Q=32.00(\mathrm{p}<0.0001)$

$F^{2}=90.6 \%$

Biasindicators: Begg-Mazumdar: Kendall'stau $=-0.33 ; p=0.33$;

Egger: bias $=-1.41(95 \% \mathrm{Cl}-20.3-17.5) ; \mathrm{p}=0.77$ represented by HT in those cases with favorable prognostic features [13].

$\mathrm{mTOR}$ is a serine/threonine protein kinase downstream PI3K/Akt pathway, which controls cell growth, proliferation, survival, metabolism and angiogenesis and is involved in cancer development [14]. Rapamycin (sirolimus) and its analogs CCI-779 (temsirolimus), RAD001 (everolimus) and AP23573 inhibit mTOR activation, that is often involved in mechanisms of anticancer drug resistance [15]. On this basis, a potential strategy to antagonize cellular HT escape is to target mTOR or its functionally related signalling proteins, such as PI3K, PTEN, AKT [16].

In preclinical studies, temsirolimus inhibited mTOR and restored sensitivity to tamoxifen primarily through induction of apoptosis, suggesting that AKTinduced resistance to tamoxifen may be mediated by escape from cell death [17]. Preliminary studies showed that everolimus reversed AKT-mediated cell resistance and restored responsiveness to letrozole
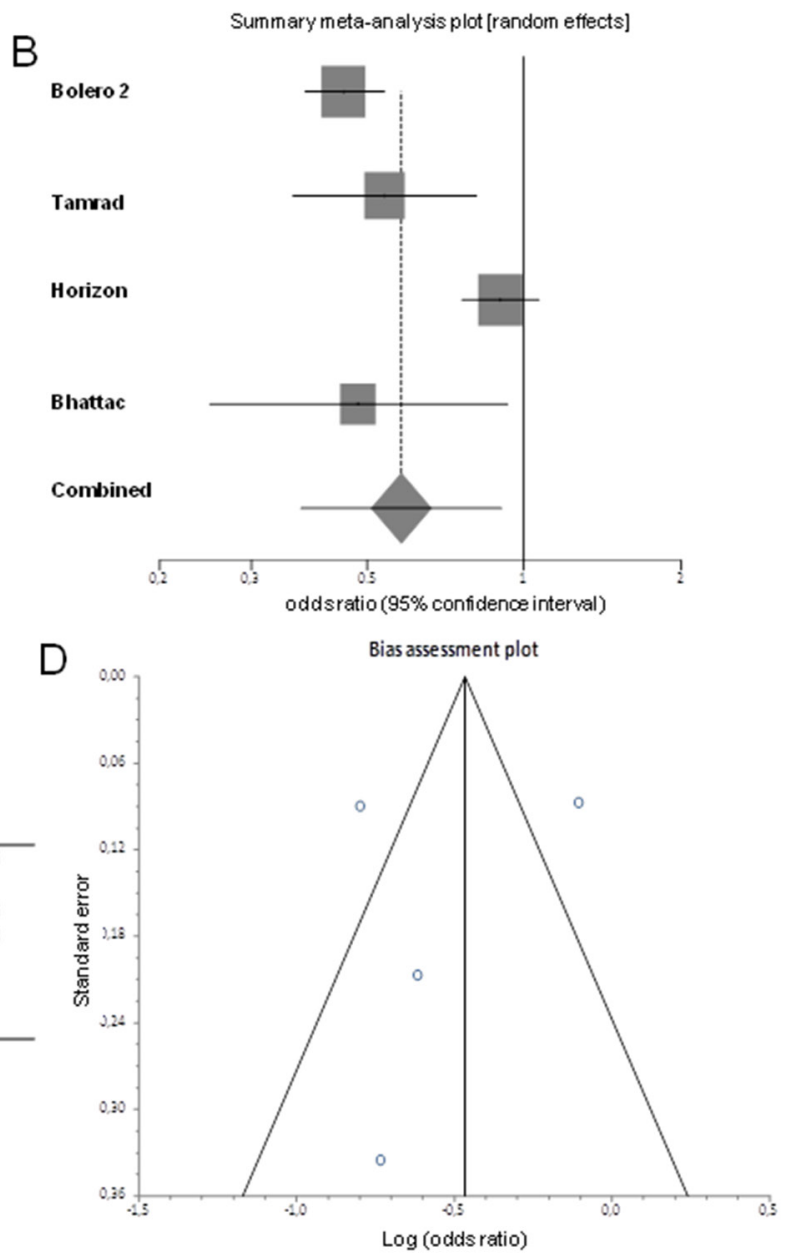

Figure 2: Forest plots of progression free survival/time to progression and Funnel plot for publication bias. Pooled HR with 95\% CI were generated with fixed and random effects models and the respective forest plots A-B are reported up in the figure. Cochran's Q and I ${ }^{2}$ index tests for detecting heterogeneity, Begg-Mazumdar and Egger tests for disclosing publication bias were performed C. A funnel plot $\mathbf{D}$ was drawn for checking bias. 
or fulvestrant [18]. On these bases, there is a strong rationale for combination of anti-estrogen drugs with rapamycin analogs. However, tumors with high PI3KAKT-mTOR activity are heterogeneous in response and novel biomarkers are required to identify breast cancer subtypes that could benefit from the combination [19]. The results of current clinical trials appear, as first impact, to confirm the preclinical findings. The addition of a mTOR-I to HT resulted in improved TTP in TAMRAD trial (HR: 0.54; 95\% CI 0.36-0.81) [5], improved PFS in Bhattacharyya trial (HR: 0.48; 95\% CI 0.25-0.93; $p=0.0028$ ) [12], improved PFS in BOLERO-2 trial (HR 0.45; 95\% CI 0.38-0.54; $p<0.0001$ ) [7], while same results were not demonstrated for temsirolimus in the large HORIZON trial population, that showed similar PFS in both arms (HR 0.90; 95\% CI 0.76-1.07; $p=0.25$ ). The results of HORIZON study can be explained by exclusion of patients pretreated with AI, who could not be considered HT-resistant [11].

The benefits derived from individual trials have been significantly maintained in the whole meta-analysis population (pooled HR for PFS/TTP:

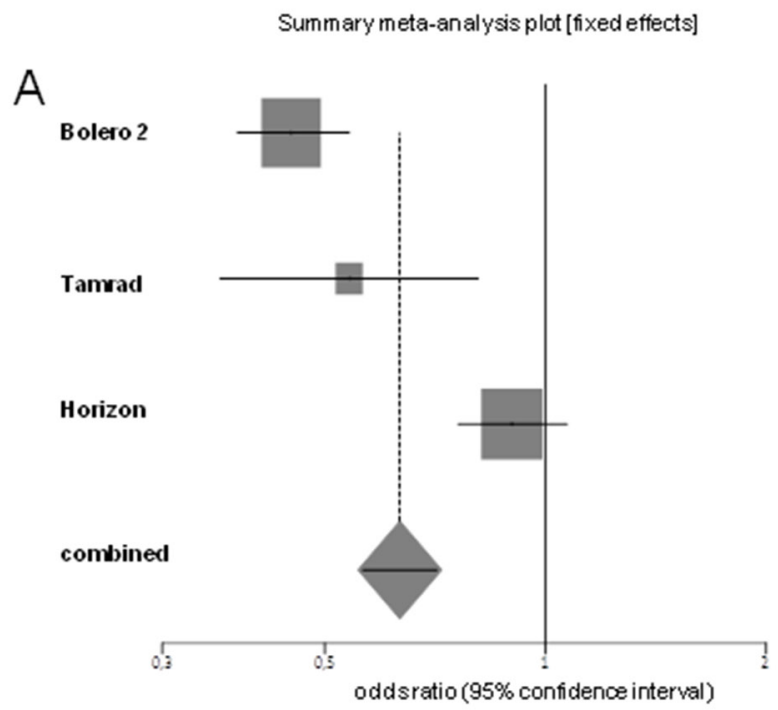

0.62; 95\% CI 0.55-0.70; $p<0.0001)$. However, study heterogeneity for PFS/TTP was found and could be also demonstrated when Bhattacharyya trial was removed from the analysis.

For the OS endpoint, TAMRAD trial moved the balance in favor of combination (HR 0.45 ; 95\% CI $0.24-$ $0.81 ; p=0.007)$, taking into account that the other trials were not significant on this endpoint $[5,8,11,12]$. In meta-analysis pooled OS was significantly prolonged in the mTOR-I+HT arm, with a $16 \%$ reduction of risk of death $(p=0.04)$; data were homogeneus. Bhattacharyya trial did not report OS data.

ORR was higher in experimental arm in BOLERO-2 trial $(12.6 \%$ versus $1.7 \% ; p<0.0001)$ [7], while it was similar in both arms in TAMRAD (14\% versus $13 \%)$ [5] and HORIZON (27\% versus 27\%) [11]. Pooled RR for ORR, performed without Bhattacharyya trial, was $0.88(p<0.0001)$ in favor of the experimental arm. Heterogeneity was not significant.

AEs, in particular those of grade 3-4, were mostly increased in mTOR-I+HT arm both in single trials $[5,8$, $9,11,12]$ and in meta-analysis pooled data, even for cases

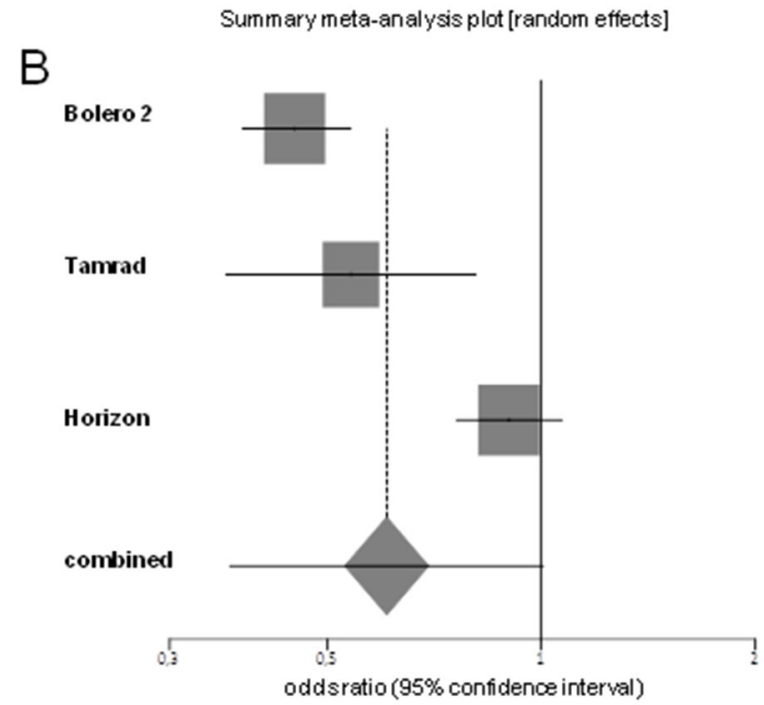

\begin{tabular}{|c|c|c|c|c|}
\hline Study & HR $(95 \% \mathrm{Cl})$ & Z & $\begin{array}{l}\text { Weight \% } \\
\text { (fixed) }\end{array}$ & $\begin{array}{l}\text { Weight \% } \\
\text { (random) }\end{array}$ \\
\hline Bolero 2 & $0.45(0.38-0.54)$ & & 44.585207 & 35.105155 \\
\hline Tamrad & $0.54(0.36-0.81)$ & & 8.371863 & 29.712568 \\
\hline Horizon & $0.90(0.76-1.07)$ & & 47.04293 & 35.182277 \\
\hline Total (fixed) & $0.63(0.56-0.71)$ & $-7.63(p<0.0001)$ & 100 & \\
\hline Total (random) & $0.60(0.36-1.00)$ & $-1.93(p=0.05)$ & & 100 \\
\hline
\end{tabular}

Figure 3: Forest plots of progression free survival/time to progression without Bhattacharyya trial. Pooled HR with $95 \%$ CI were generated with fixed and random effects models and the respective forest plots A-B are reported. Cochran's Q and I² index tests for detecting heterogeneity were performed $\mathbf{C}$. 
of rare toxicity-related deaths. The most common AEs that led to dose interruptions/reductions, such as fatigue, pneumonitis and dyspnea [5, 8, 9, 11, 12], were also referred to the combination arm. The sirolimus study was not included because data on toxicity were not available.

All together, these results suggest that combination therapy with mTOR-I improves the efficacy of HT in metastatic luminal breast cancer patients and impacts on survival. The reported heterogeneity relies on various factors, for example heterogeneous study populations (HT naïve in HORIZON, in failure after previous HT in the other studies) and imbalance in post-study treatment lines. BOLERO-2 trial and HORIZON trial sample sizes were weighted on PFS primary endpoint and not on OS, while TAMRAD trial sample size was based on clinical benefit rate as primary endpoint. In TAMRAD trial and in Bhattacharyya trial only TTP was selected as endpoint. Finally, in Bhattacharyya trial, primary endpoints were ORR and TTP. Many potential limitations can affect our results, because differences in the design, patients characteristics, methodological quality and execution of the single primary studies could not be overcame. An additional important point is that, even if results were obtained from randomized clinical trials, we did not

Summary meta-analysis plot [fixed effects]

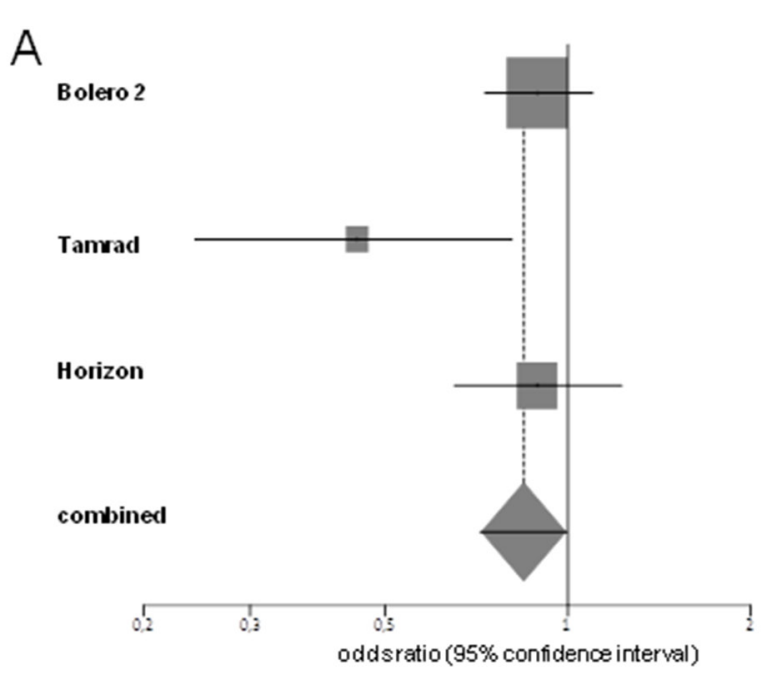

handle individual patients data, but whole populations data were extracted from the available reports. Moreover not all outcomes were reported in all studies and our work was retrospective. Risks of selection or publication biases, due to selection of studies that report dramatic effects and to exclusion of studies that report smaller effect sizes, have not been shown in our meta-analysis, except for PFS/TTP, where no funnel plot asymmetry was detected. Furthermore, as previously described, we repeated the analysis for PFS/TTP excluding one of the original studies (Bhattacharyya trial) to determine if the overall conclusions have been influenced by study selection.

In the light of our results, we can conclude that mTOR-I offer a benefit as a class-effect, even though the differences among drugs investigated in the individual trials and among population characteristics should not be overlooked.

The use of mTOR-I offers a benefit that must be weighted in the clinical scenario of metastatic luminal breast cancer. In palliative setting, the gain of few months in PFS/TTP, showed in our work, should be balanced with the patient quality of life, which rather could be affected by side effects of mTOR-I treatment.

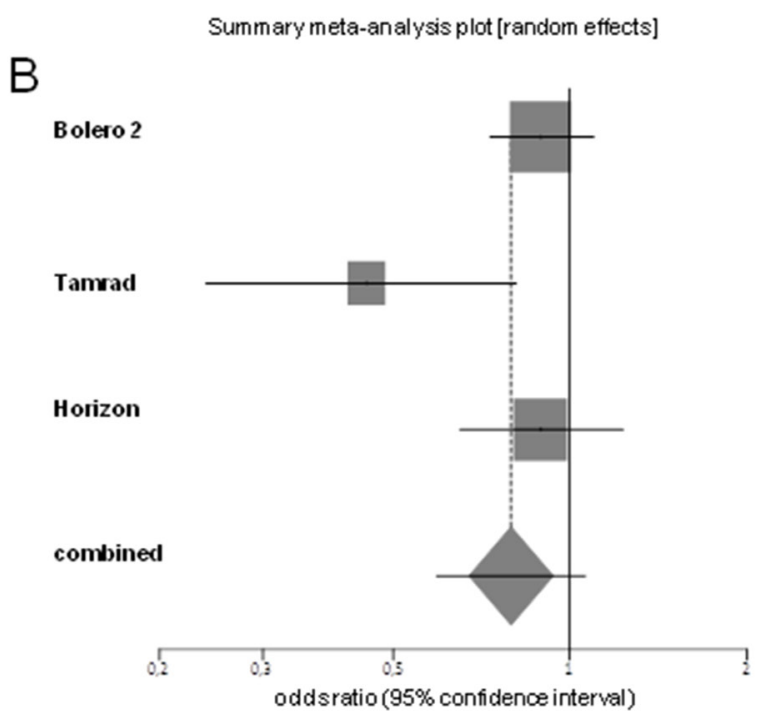

\begin{tabular}{|c|c|c|c|c|}
\hline Study & HR $(95 \%$ CI $)$ & Z & $\begin{array}{l}\text { Weight \% } \\
\text { (fixed) }\end{array}$ & $\begin{array}{l}\text { Weight \% } \\
\text { (random) }\end{array}$ \\
\hline Bolero2 & $0.89(0.73-1.10)$ & & 65.491981 & 47.486791 \\
\hline Tamrad & $0.45(0.24-0.81)$ & & 7.441332 & 16.840328 \\
\hline Horizon & $0.89(0.65-1.23)$ & & 27.066687 & 35.672881 \\
\hline Total (fixed) & $0.84(0.71-0.99)$ & $-1.97(p=0.04)$ & 100 & \\
\hline Total (random) & $0.79(0.59-1.06)$ & $-1.55(p=0.12)$ & & 100 \\
\hline
\end{tabular}

Figure 4: Forest plots of overall survival. Pooled HR with 95\% CI were generated with fixed and random effects models and the respective forest plots are reported A-B. Cochran's Q and I² index tests for detecting heterogeneity were performed $\mathbf{C}$. 
In particular, in asymptomatic luminal patients with good prognosis, the combination treatment should be comparable to the HT alone in terms of toxicity to ensure the maintenance of good clinical conditions. Cost-effectiveness analysis demonstrated conflicting results on everolimus plus exemestane compared to exemestane alone in BOLERO-2 [20]. Longterm results and analysis of post-marketing studies are indeed needed to finally address this important issue. A further point is the inclusion of mTOR-I in the therapeutic algorithm for patient continuum of care. At present mTOR-I have been investigated in neoadjuvant setting, with limited benefits [21], while new studies are ongoing in the adjuvant setting [22-23]. In metastatic disease, the trials included in our metaanalysis allocated the combination treatment in HT naïve or in patients who failed HT. Evidence in favor of combined HT plus mTOR-I rather than chemotherapy with or without biological agents, such as bevacizumab in HER-2 negative breast cancer, in first line or in subsequent lines, is not still available. This comparison is indeed very difficult, due to selection bias in favor of chemotherapy for patients with more aggressive disease. However, although chemotherapy is the mainstay in patients at risk of visceral crisis, BOLERO-2 subgroup analysis showed that patients with visceral metastasis can indeed benefit from everolimus and exemestane combination [24].

Finally, the role of novel agents that can potentiate mTOR blockade, is under investigation. The combination of PI3K and CDK4/6 inhibitors demonstrated promising data on apoptosis induction, due to sensitization of ER-positive cells to CDK4/6 inhibition by suppressing cyclin D1 expression [25]. Targeting the PI3K pathway, such as by dual inhibitors of PI3K and mTOR, is another strategy presently under investigation [26].

We think that our study provides an important proof-of-concept that interference with mTOR is a crucial biologic mechanism regulating hormone sensitivity in luminal breast cancer, that must be weighted in the clinical scenario and further efforts are necessary in order to correctly allocate these agents in the patient continuum of care.

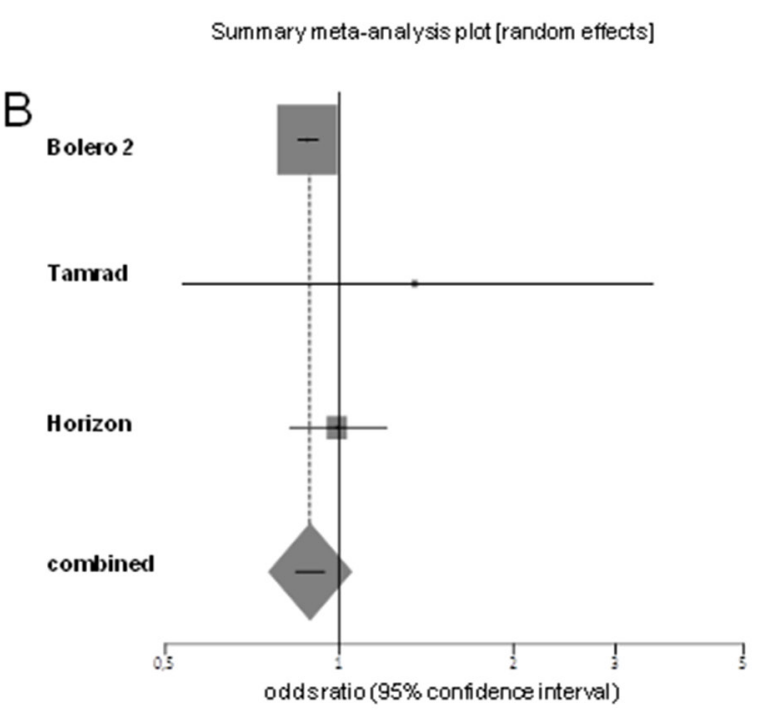

\begin{tabular}{|c|c|c|c|c|}
\hline Study & $\operatorname{RR}(95 \% \mathrm{Cl})$ & z & $\begin{array}{l}\text { Weight \% } \\
\text { (fixed) }\end{array}$ & $\begin{array}{l}\text { Weight \% } \\
\text { (random) }\end{array}$ \\
\hline Bolero2 & $0.88(0.85-0.92)$ & & 95.868118 & 91.277928 \\
\hline Tamrad & $1.35(0.52-3.49)$ & & 0.165646 & 0.367346 \\
\hline Horizon & $0.99(0.82-1.21)$ & & 3.966236 & 8.354727 \\
\hline Total (fixed) & $0.88(0.85-0.91)$ & $-6.19(p<0.0001)$ & 100 & \\
\hline Total (random) & $0.89(0.84-0.94)$ & $-3.95(p<0.0001)$ & & 100 \\
\hline
\end{tabular}

Figure 5: Forest plots of objective response rate. Pooled RR with $95 \%$ CI were generated with fixed and random effects models and the respective forest plots are reported A-B. Cochran's Q and I ${ }^{2}$ index tests for detecting heterogeneity were performed $\mathbf{C}$. 
Table 2: Meta-analysis results: safety

\begin{tabular}{|c|c|c|c|c|c|c|c|c|}
\hline \multicolumn{9}{|c|}{ Meta-analysis of any grade adverse events. } \\
\hline & $\begin{array}{l}\text { Fixed-effects } \\
\text { RR }(95 \% \text { CI })\end{array}$ & $\mathbf{Z}$ & $\mathbf{P}$ & $\begin{array}{c}\text { Heterogeneity } \\
\text { Cochran's Q (P) }\end{array}$ & $\begin{array}{c}I^{2} \text { INDEX } \\
(\%)\end{array}$ & $\begin{array}{c}\text { Random-effects } \\
\text { RR (95\%CI) }\end{array}$ & $\mathbf{Z}$ & $\mathbf{P}$ \\
\hline \multirow[t]{2}{*}{ Asthenia } & 0.989 & -2.48 & 0.01 & 4.90 & 79.6 & 0.961 & -1.06 & 0.28 \\
\hline & $(0.981-0.997)$ & & & $(0.02)$ & & $(0.895-1.033)$ & & \\
\hline \multirow[t]{2}{*}{ Fatigue } & 0.97 & -2.63 & 0.008 & 4.06 & 75.4 & 0.80 & -0.85 & 0.39 \\
\hline & $(0.96-0.99)$ & & & $(0.04)$ & & $(0.48-1.32)$ & & \\
\hline \multirow[t]{2}{*}{ Diarrhea } & 0.85 & -6.86 & $<0.0001$ & 3.76 & 73.4 & 0.78 & -2.10 & 0.03 \\
\hline & $(0.81-0.89)$ & & & $(0.05)$ & & $(0.62-0.98)$ & & \\
\hline \multirow[t]{2}{*}{ Stomatitis } & 0.95 & -6.33 & $<0.0001$ & 37.28 & 94.6 & 0.87 & -2.49 & 0.01 \\
\hline & $(0.94-0.96)$ & & & $(<0.0001)$ & & $(0.78-0.97)$ & & \\
\hline \multirow[t]{2}{*}{ Pneumonitis } & 0.93 & -5.97 & $<0.0001$ & 1.65 & 39.7 & 0.92 & -2.21 & 0.02 \\
\hline & $(0.91-0.95)$ & & & $(0.19)$ & & $(0.85-0.99)$ & & \\
\hline \multirow[t]{2}{*}{ Hyperglycemia } & 0.996 & -1.95 & 0.05 & 21.16 & 95.3 & 0.95 & -1.02 & 0.30 \\
\hline & $(0.992-1.00)$ & & & $(<0.0001)$ & & $(0.87-1.04)$ & & \\
\hline \multirow[t]{2}{*}{ Infection } & 0.95 & -2.44 & 0.01 & 2.23 & 55.3 & 0.91 & -1.12 & 0.25 \\
\hline & $(0.92-0.99)$ & & & $(0.13)$ & & $(0.77-1.07)$ & & \\
\hline \multirow[t]{2}{*}{ Rash } & 0.97 & -5.49 & $<0.0001$ & 41.90 & 95.2 & 0.86 & -2.30 & 0.02 \\
\hline & $(0.96-0.98)$ & & & $(<0.0001)$ & & $(0.77-0.97)$ & & \\
\hline \multirow[t]{2}{*}{ Dyspnea } & 0.96 & -4.22 & $<0.0001$ & 0.17 & 0 & 0.96 & -4.22 & $<0.0001$ \\
\hline & $(0.95-0.98)$ & & & $(0.67)$ & & $(0.95-0.98)$ & & \\
\hline \multicolumn{9}{|c|}{ Meta-analysis of grade 3-4 adverse events. } \\
\hline \multirow[t]{2}{*}{ Asthenia } & 0.994 & -1.97 & 0.04 & 0.22 & 0 & 0.994 & -1.97 & 0.04 \\
\hline & $(0.989-0.999)$ & & & $(0.63)$ & & $(0.989-0.999)$ & & \\
\hline \multirow[t]{2}{*}{ Fatigue } & 0.991 & -2.12 & 0.03 & 1.06 & 6.3 & 0.99 & -0.64 & 0.52 \\
\hline & $(0.983-0.999)$ & & & $(0.30)$ & & $(0.97-1.01)$ & & \\
\hline \multirow[t]{2}{*}{ Diarrhea } & 0.98 & -2.79 & 0.005 & 0 & 0 & 0.98 & -2.79 & 0.005 \\
\hline & $(0.96-0.99)$ & & & $(>0.99)$ & & $(0.96-0.99)$ & & \\
\hline \multirow[t]{2}{*}{ Stomatitis } & 0.991 & -2.22 & 0.02 & 6.33 & 68.4 & 0.98 & -1.27 & 0.20 \\
\hline & $(0.983-0.998)$ & & & $(0.04)$ & & $(0.97-1.00)$ & & \\
\hline \multirow[t]{2}{*}{ Pneumonitis } & 0.98 & -2.43 & 0.01 & 0.80 & 0 & 0.98 & -2.43 & 0.01 \\
\hline & $(0.96-0.99)$ & & & $(0.37)$ & & $(0.96-0.99)$ & & \\
\hline \multirow[t]{2}{*}{ Hyperglycemia } & 0.996 & -1.99 & 0.04 & 6.56 & 84.8 & 0.98 & -1.08 & 0.27 \\
\hline & $(0.992-0.999)$ & & & $(0.01)$ & & $(0.95-1.01)$ & & \\
\hline \multirow[t]{2}{*}{ Infection } & 0.98 & -1.69 & 0.09 & 0.16 & 0 & 0.98 & -1.69 & 0.09 \\
\hline & $(0.97-1.001)$ & & & $(0.68)$ & & $(0.97-1.001)$ & & \\
\hline \multirow[t]{2}{*}{ Rash } & 0.988 & -3.95 & $<0.0001$ & 5.55 & 64 & 0.98 & -2.26 & 0.02 \\
\hline & $(0.982-0.994)$ & & & $(0.06)$ & & $(0.97-0.99)$ & & \\
\hline \multirow[t]{2}{*}{ Dyspnea } & 0.98 & -3.90 & $<0.0001$ & 0.77 & 0 & 0.98 & -3.90 & $<0.0001$ \\
\hline & $(0.97-0.99)$ & & & $(0.37)$ & & $(0.97-0.99)$ & & \\
\hline
\end{tabular}




\section{PATIENTS AND METHODS}

We provided to search trials on public databases and the analysis was performed in accordance with the Preferred Reporting Items for Systematic Reviews and Meta-analyses (PRISMA) guidelines (PRISMA 2009 checklist) [27]. No study involving human participants and requiring ethics committee approval in accordance to the Declaration of Helsinki and its subsequent revisions was conducted during our investigation.

\section{Eligibility criteria}

\section{Inclusion criteria}

The studies concerned the diagnosis of metastatic luminal HER2-negative breast cancer. The trials might be randomized, prospective and controlled. We considered congress abstracts if containing sufficient information about the study design, the patient characteristics, outcomes and toxicity. Patients in the experimental arm received mTOR-I plus HT. Patients in the control arm received only HT.

\section{Exclusion criteria}

No comparative studies, no randomized clinical trials, studies that not involved our target drugs, studies with no comparable endpoints, other than oral administration and languages other than English were excluded.

\section{Information sourches and search strategy}

Public databases (PubMed, Embase, Central Registry of Controlled Trials of the Cochrane Library) full texts and abstracts were interrogated for the 2005-2015 time frame. Google academic search (including also meeting abstracts) was performed to track relevant references, too. The search included the following keywords: "breast" AND ("cancer" OR "cancers" OR "carcinoma" OR "carcinomas") AND "phase II" OR "phase III" AND "mTOR" AND ("inhibitor" OR "everolimus" OR "RAD001" OR "rapamycin" OR "sirolimus" OR "PI-103" OR “temsirolimus" OR “torisel”).

\section{Study selection and data collection process}

The studies were examined independently by two investigators in order to select eligible studies (MSR, TG). Numerous selected variables were extracted and evaluated, such as the number of enrolled patients, year of publication, the treatment program and activity and efficacy endpoints. The data concerning the occurrence of toxicity were obtained from the safety profile of each study. Any discrepancy was resolved by an arbitrator (PT). We considered all patients for PFS/TTP, OS, ORR and toxicity, with any grade and grade 3-4 AEs.

\section{Summary measures and statistical analysis}

HR with their corresponding 95\% CI for PFS/TTP and OS, RR for ORR and for any grade and grade 3-4 AEs were compared in the two groups: mTOR-I+HT versus HT alone. The meta-analysis was performed with an alpha error of 0.05 and the $p$ value $<0.05$ was considered to be significant. A fixed-effects model was chosen and analysis by random-effects model was added to generate a more conservative estimate when the heterogeneity at Cochran's $\mathrm{Q}$ test showed significant difference $(p<0.05)$. In addition to Cochran's Q, the $\mathrm{I}^{2}$ index has been performed to quantify the degree of heterogeneity and to give a better measure of the inter-trials consistency. For higher values of $\mathrm{I}^{2}$ index, heterogeneity is more enhanced $\left(\mathrm{I}^{2}\right.$ index of $25 \%, 50 \%$ and $75 \%$ corresponds to low, medium and high heterogeneity, respectively). Begg and Egger tests were performed to evaluate potential publication biases and they were considered significant when the $p$-value resulted $<0.05$. Forest plots are reported to display the meta-analysis results and a funnel plot was drawn to detect biases when we could evaluate $>3$ trials (pooled HR for PFS/TTP). We used Stats Direct 3 software for statistical analysis.

\section{CONFLICTS OF INTEREST}

The authors have no support or funding to report and declare no conflicts of interest.

The authors confirm that neither the submitted manuscript nor any similar manuscript, in whole or in part, is under consideration, in press, published, or reported elsewhere.

\section{REFERENCES}

1. Perou CM, Sorlie T, Eisen MB, van de Rijn M, Jeffrey SS, Rees CA, Pollack JR, Ross DT, Johnsen H, Akslen LA, Fluge O, Pergamenschikov A, Williams C, et al. Molecular portraits of human breast tumours. Nature. 2000 Aug 17; 406: 747-52.

2. Sorlie T, Perou CM, Tibshirani R, Aas T, Geisler S, Johnsen $\mathrm{H}$, Hastie T, Eisen MB, van de Rijn M, Jeffrey SS, Thorsen T, Quist H, Matese JC, et al. Gene expression patterns of breast carcinomas distinguish tumor subclasses with clinical implications. Proc Natl Acad Sci USA. 2001 Sep 11; 98: 10869-74.

3. Jacinto E, Hall MN. Tor signalling in bugs, brain and brawn. Nat Rev Mol Cell Biol. 2003; 4: 117-26.

4. Cash H, Shah S, Moore E, Caruso A, Uppaluri R, Van Waes C, Allen C. mTOR and MEK1/2 inhibition differentially modulate tumor growth and the immune microenvironment in syngeneic models of oral cavity cancer. Oncotarget. 2015 Oct 22. Doi: 10.18632/oncotarget.5063. 
5. Bachelot T, Bourgier C, Cropet C, Ray-Coquard I, Ferrero JM, Freyer G, Abadie-Lacourtoisie S, Eymard JC, Debled M, Spaeth D, Legouffe E, Allouache D, El Kouri C, et al. Randomized Phase II trial of everolimus in combination with tamoxifen in patients with hormone receptor-positive, human epidermal growth factor receptor 2-negative metastatic breast cancer with prior exposure to aromatase inhibitors: a GINECO study. J Clin Oncol. 2012 Aug 1; 30: 271824. Epub 2012 May 7.

6. Baselga J, Campone M, Piccart M, Burris HA 3rd, Rugo HS, Sahmoud T, Noguchi S, Gnant M, Pritchard KI, Lebrun F, Beck JT, Ito Y, Yardley D, et al. Everolimus in postmenopausal hormone receptor-positive advanced breast cancer. N Engl J Med. 2012 Feb 9; 366: 520-29.

7. Yardley DA, Noguchi S, Pritchard KI, Burris HA, Baselga J, Gnant M, Hortobagyi GN, Campone M, Pistilli B, Piccart M, Melichar B, Petrakova K, Arena FP, et al. Everolimus plus exemestane in postmenopausal patients with $\mathrm{HR}+$ breast cancer: BOLERO-2 final progression free survival analysis. Adv Ther. 2013; 30: 870-84.

8. Piccart M, Hortobagyi GN, Campone M, Pritchard KI, Lebrun F, Ito Y, Noguchi S, Perez A, Rugo HS, Deleu I, Burris HA 3rd, Provencher L, Neven P, et al. Everolimus plus exemestane for hormone-receptor-positive, human epidermal growth factor receptor-2-negative advanced breast cancer: overall survival results from BOLERO-2. Ann Oncol. 2014 Dec; 25: 2357-62.

9. Rugo HS, Pritchard KI, Gnant M, Noguchi S, Piccart M, Hortobagyi G, Baselga J, Perez A, Geberth M, Csoszi T, Chouinard E, Srimuninnimit V, Puttawibul P, et al. Incidence and time course of everolimus-related adverse events in postmenopausal women with hormone receptorpositive advanced breast cancer: insights from BOLERO-2. Ann Oncol. 2014 Apr; 25: 808-15.

10. Carpenter JT, Rochè H, Campone M, Colomer R, JagielloGruszfeld A, Moore L, D’Amore M, Kong S, Boni J, Baselga J. Randomized 3-arm, phase 2 study of temsirolimus (CCI-779) in combination with letrozole in postmenopausal women with locally advanced or metastatic breast cancer. J Clin Oncol (Meeting Abstracts). 2005 June; 23: 564.

11. Wolff AC, Lazar AA, Bondarenko I, Garin AM, Brincat S, Chow L, Sun Y, Neskovic-Konstantinovic Z, Guimaraes RC, Fumoleau P, Chan A, Hachemi S, Strahs A, et al. Randomized phase III placebo-controlled trial of letrozole plus oral temsirolimus as first-line endocrine therapy in postmenopausal women with locally advanced or metastatic breast cancer. J Clin Oncol. 2013 Jan 10; 31: 195-202.

12. Bhattacharyya GN, Biswas J, Singh JH, Sing $M$, Govindbabu K, Ranade AA, Malhotra H, Parikh PM, Shahid T, Basu S. Reversal of tamoxifen resistance (hormone resistance) by addition of sirolimus (mTOR inhibitor) in metastatic breast cancer. Eur J Cancer. 2011; 47: 9.
13. Howell SJ. Advances in the treatment of luminal breast cancer. Curr Opin Obstet Gynecol. 2013 Feb; 25: 49-54.

14. Abraham RT. Identification of TOR signaling complexes: more TORC for the cell growth engine. Cell. 2002 Oct 4; 111: 9-12.

15. Jiang BH, Liu LZ. Role of mTOR in anticancer drug resistance: perspectives for improved drug treatment. Drug Resist Updat. 2008 June; 11: 63-76.

16. Granville CA, Memmott RM, Gills JJ, Dennis PA. Handicapping the race to develop inhibitors of the phosphoinositide 3-kinase/Akt/mammalian target of rapamycin pathway. Clin Cancer Res. 2006 Feb 1; 12: 679-89.

17. DeGraffenried LA, Friedrichs WE, Russell DH, Donzis EJ, Middleton AK, Silva JM, Roth RA, Hidalgo M. Inhibition of mTOR activity restores tamoxifen response in breast cancer cells with aberrant Akt Activity. Clin Cancer Res. 2004 Dec 1; 10: 8059-67.

18. Beeram M, Tan Q-TN, Tekmal RR, Russell D, Middleton A, deGraffenried LA. Akt- induced endocrine therapy resistance is reversed by inhibition of mTOR signaling. Ann Oncol. 2007; 18: 1323-28.

19. Polo ML, Riggio M, May M, Rodriguez MJ, Perrone MC, Stallings-Mann M, Kaen D, Frost M, Goetz M, Boughey J, Lanari C, Radisky D, Novaro V. Activation of PI3K/Akt/ mTOR signaling in the tumor stroma drives endocrine therapy-dependent breast tumor regression. Oncotarget. 2015 Sep 8; 6: 22081-97. doi: 10.18632/oncotarget.4203.

20. Diaby V, Adunlin G, Zeichner SB, Avancha K, Lopes G, Gluck S, Montero AJ. Cost-effectiveness analysis of everolimus plus exemestane versus exemestane alone for treatment of hormone receptor positive metastatic breast cancer. Breast Cancer Res Treat. 2014 Sep; 147: 433-41.

21. Baselga J, Semiglazov V, van Dam P, Manikhas A, Bellet M, Mayordomo J, Campone M, Kubista E, Greil R, Bianchi G, Steinseifer J, Molloy B, Tokaji E et al. Phase II randomized study of neoadjuvant everolimus plus letrozole compared with placebo plus letrozole in patients with estrogen receptor-positive breast cancer. J Clin Oncol. 2009 Jun 1; 27: 2630-7.

22. Bachelot TD, Chabaud S, Martin AL, Lemonnier J, Campone M, Andre F. UNIRAD: Multicenter, doubleblind, phase III study of everolimus plus ongoing adjuvant therapy in ER+, HER2- breast cancer. J Clin Oncol. 2013; 31: abstr TPS653.

23. Chavez-Mac Gregor M, Barlow WE, Pusztai L, Goetz MP, Rastogi P, Ganz PA, Mamounas EP, Paik S, Bandos H, Gralow J, Lew DL, Hortobagyi GN. Phase III randomized, placebo-controlled clinical trial evaluating the use of adjuvant endocrine therapy $+/$ - one year of everolimus in patients with high-risk, hormone receptor (HR) positive and HER2-negative breast cancer (BC): SWOG/NRG/ Alliance S1207 (NCT01674140). J Clin Oncol. 2015; 33: abstr TPS637. 
24. Campone M, Bachelot T, Gnant M, Deleu I, Rugo HS, Pistilli B, Noguchi S, Shtivelband M, Pritchard KI, Provencher L, Burris III HA, Hart L, Melichar B et al. Effect of visceral metastases on the efficacy and safety of everolimus in postmenopausal women with advanced breast cancer: Subgroup analysis from the BOLERO-2 study. Eur J Cancer. 2013 Aug; 49: 2621-32.

25. Herrera-Abreu MT, Asghar US, Elliot R, Pearson A, Nannini MA, Young A, Sampath D, Dowsett M, Martin LA, Turner N. PI3 kinase/mTOR inhibition increases sensitivity of ER positive breast cancers to CDK4/6 inhibition by blocking cell cycle re-entry driven by cyclin D1 and inducing apoptosis. Ann Oncol 2015; 26: iii29-iii30.

26. Sabbah DA, Brattain MG, Zhong H. Dual inhibitors of $\mathrm{PI} 3 \mathrm{~K} / \mathrm{mTOR}$ or mTOR-selective inhibitors: which way shall we go? Curr Med Chem. 2011. 18: 5528-44.

27. Moher D, Liberati A, Tetzlaff J, Altman DG and the PRISMA Group. Preferred reporting items for systematic reviews and meta-analyses: the PRISMA statement. PLoS Med. 2009 Jul; 6: e1000097. 